\title{
A sintomas motores mais comuns em um grupo de parkinsonianos de um centro de reabilitação no interior do estado do Rio de Janeiro
}

\section{Maira Pedrosa dos Santos}

Fisioterapeuta - Centro Universitário de Barra Mansa - UBM.

ORCID: https://orcid.org/0000-0002-6798-2606

E-mail: mairaa bm@hotmail.com

\section{Juliana de Oliveira Souza}

Mestranda em Engenharia Biomédica - Universidade do Vale do Paraíba - UNIVAP. Fisioterapeuta da Prefeitura Municipal de Santa Rita de Jacutinga - MG.

ORCID: https://orcid.org/0000-0002-0971-8241

E-mail: jufisio souza@yahoo.com.br

\section{Sandra São Thiago da Costa Pereira}

Mestre em Engenharia Biomédica - Universidade Estadual Paulista UNESP. Docente no Centro Universitário de Barra Mansa - UBM.

ORCID: https://orcid.org/0000-0002-1888-863X

E-mail: sandrasaothiago@hotmail.com.

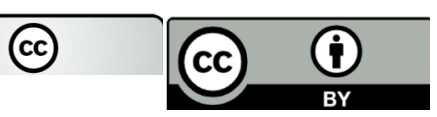




\section{Resumo}

Os sintomas motores da Doença de Parkinson (DP) geram incapacidade funcional. Objetivos: Identificar os sintomas motores mais comuns em um grupo de Parkinsonianos de um Centro de Reabilitação no interior do estado do Rio de Janeiro. Métodos: Estudo de caráter transversal observacional descritivo, com 30 parkinsonianos, de ambos os gêneros, com média de idade de 64 anos. Realizouse entrevista individual transcrita, com questionário próprio adaptado de instrumentos consagrados na literatura. Os dados foram analisados através de frequência e percentual com auxílio do programa Microsoft ${ }^{\circledR}$ Excel. Resultados: A maioria dos voluntários apresentava bradicinesia (87\%), os outros sintomas foram instabilidade postural (73\%), micrografia (70\%), marcha com passos curtos $(70 \%)$, rigidez muscular $(63 \%)$, tremor $(63 \%)$, câimbra $(60 \%)$, congelamento (freezing) (57\%), redução ou ausência do balanço dos braços $(47 \%)$, distonia (43\%), hipofonia (43\%) e discinesia (33\%). Conclusão: Diversos são os comprometimentos motores dos voluntários, sendo a bradicinesia o mais comum entre eles.

Palavras-chave: Doença de Parkinson; Sinais e sintomas; Doenças dos gânglios da base; Envelhecimento; Dopamina.

\section{Abstract}

Motor symptoms of Parkinson's disease (PD) cause functional disability. Objectives: To identify the most common motor symptoms in a group of Parkinsonians at a Rehabilitation Center in the interior of the state of Rio de Janeiro. Methods: A descriptive observational cross-sectional study, with 30 parkinsonians, of both genders, with a mean age of 64 years. A transcribed individual interview was conducted, with a questionnaire adapted from instruments established in the literature. Data were analyzed using frequency and percentage with the aid of the Microsoft ${ }^{\circledR}$ Excel program. Results: Most volunteers had bradykinesia (87\%), the other symptoms were postural instability (73\%), micrograph (70\%), walking with short steps (70\%), muscle stiffness (63\%), tremor $(63 \%)$, cramps $(60 \%)$, freezing $(57 \%)$, reduction or absence of am balance (47\%), dystonia (43\%), hypophonia (43\%) and dyskinesia (33\%). Conclusion: Several are the motor impairments of the volunteers, with bradykinesia being the most common among them.

Keywords: Parkinson's Disease; Signals and symptons; Basal ganglia diseases; Aging. Dopamine. 


\section{Introdução}

A longevidade é uma das grandes conquistas do século passado, um fenômeno mundial que, juntamente com a queda da fecundidade, ocasiona um drástico envelhecimento na população do planeta. Esse processo começou em épocas distintas, em países diferentes, e evolui em proporções variadas. No Brasil, os efeitos são ainda maiores em razão do pequeno período de tempo em que vem ocorrendo. Todo ano, 700 mil novos idosos são incorporados à população brasileira (VERAS, 2016). No ano de 1960, o número de idosos passou de 3 milhões; aumentando para 7 milhões em 1975; e 14 milhões em 2002; podendo alcançar 32 milhões em 2020 (VERAS; OLIVEIRA, 2018).

Esse novo cenário provocou um desenvolvimento notável de doenças crônicas degenerativas (FARIA; LIMA; PEREIRA-SILVA, 2019), dentre elas a Doença de Parkinson (DP), uma doença neurodegenerativa progressiva crônica do sistema nervoso central (SNC), que provoca alterações motoras e não motoras (CUGUSI te al., 2015). Sem causa definida, a DP afeta as células da zona compacta da substância negra, presentes nos núcleos da base do mesencéfalo, deteriorando neurônios responsáveis pela produção de dopamina, que dentre suas funções é responsável por comandar os movimentos (GONÇALVES; ALVAREZ; ARRUDA, 2007).

A DP tem incidência na população acima de 65 anos de idade de 1 a $2 \%$ em todo o mundo e de 3,3\% no Brasil (TANG et al., 2016), sendo o segundo distúrbio mais comum após a doença de Alzheimer (ERKKINEN; GESCHWIND, 2018). A velocidade de progressão da doença e as manifestações que incapacitam o indivíduo são diferentes entre os pacientes. As características clínicas e epidemiológicas que interferem nessa evolução ainda não são bem estabelecidas e compreendidas. A idade de início da doença, a doença cronológica, o subtipo 
clínico e a presença de demência são alguns dos fatores que mais influenciam a evolução e o risco de morte (SILVA; FERNANDES; TERRA, 2014).

Os sintomas da DP são antecedidos por um extenso ciclo no qual a neurodegeneração se espalha por todo o SNC, o que se evidencia a existência da fase prodrômica, que compreende o período em que os sintomas estão presentes, entretanto, ainda não são suficientes para determinar o diagnóstico (DAYAN; BROWNER, 2017). Em 1817, o médico inglês James Parkinson descreveu as manifestações clínicas principais do que é identificado atualmente como o complexo de sintomas designado como parkinsonismo, que se manifesta por uma combinação qualquer de seis características fundamentais: tremor em repouso, rigidez, bradicinesia, postura em flexão, perda dos reflexos posturais e o fenômeno do congelamento (ROWLAND; PEDLEY, 2011). O diagnóstico clínico é fundamentado na presença de dois dos três sintomas: bradicinesia, rigidez e tremor (DAYAN; BROWNER, 2017).

Os sintomas motores geram uma elevada incapacidade funcional em seus portadores e consequentemente uma redução na qualidade de vida (QV). Portanto, todos os tratamentos existentes visam ao controle dos sintomas, objetivando manter o portador com o máximo de autonomia e independência funcional possível, proporcionando assim uma melhor QV e procurando também atrasar ao máximo a progressão da doença (SAITO, 2011).

O tratamento fisioterapêutico se mostra eficaz no tratamento da DP, com grande melhora da funcionalidade e da marcha de seus portadores (CANNING et al., 2015; SHULMAN et al., 2013). O conhecimento dos sintomas motores mais comuns na DP é fundamental para a elaboração de uma estratégia de intervenção fisioterapêutica adequada a esses indivíduos. Em virtude disso, este estudo teve como objetivo identificar os sintomas motores mais comuns em um grupo de Parkinsonianos de um Centro de Reabilitação no interior do estado do Rio de Janeiro. 


\title{
Fundamentação Teórica
}

\author{
METODOLOGIA
}

Trata-se de um estudo de caráter transversal observacional, do tipo descritivo, com 30 indivíduos com diagnóstico clínico de DP, com faixa etária entre 57 e 73 anos, de ambos os gêneros, escolhidos aleatoriamente em um Centro de Reabilitação no interior do estado do Rio de Janeiro.

Este estudo seguiu a Resolução 466/2012 e 510/2016 do Conselho Nacional de Saúde, que determina as diretrizes e normas regulamentadoras de pesquisa envolvendo seres humanos e foi aprovado pelo Comitê de Ética em Pesquisa do Centro Universitário de Barra Mansa (UBM) no dia 13 de dezembro de 2017, sob número 2.434.336. Foi garantido o anonimato aos participantes, esclarecido o objetivo e metodologia do estudo, com posterior assinatura do Termo de Consentimento Livre e Esclarecido (TCLE).

Foram incluídos indivíduos com diagnóstico clínico de DP; de ambos os gêneros, com uma adequada compreensão e competências de comunicação; que estivessem sendo assistidos no Centro de Reabilitação, medicados e com estadiamento entre 1 e 3 na Escala de Estágios de Incapacidade de Hoehn e Yahr.

Os objetivos e as condutas do estudo foram apresentados aos participantes. Eles foram submetidos a uma avaliação inicial, por meio da Escala de Estágios de Incapacidade de Hoehn e Yahr, desenvolvida em 1967 e utilizada para analisar a gravidade da DP de forma rápida e prática. Essa escala compreende cinco estágios de classificação e abrange medidas globais de sinais e sintomas que permitem classificar o indivíduo quanto ao nível de incapacidade. Os estágios de 1 a 3 indicam incapacidades leve a moderada e os estágios de 4 a 5 incapacidade grave (HOEHN; YAHR, 1967). 
A coleta de dados foi realizada no período entre fevereiro e agosto de 2018 no Centro de Reabilitação, por meio de entrevista individual transcrita, realizada pelas próprias pesquisadoras em local reservado. Utilizou-se um questionário próprio adaptado de instrumentos consagrados na literatura como: a Escala de Webster (Parkinson's Disease Rating Scale), criada em 1968, composta de 10 itens que avaliam bradicinesia, postura, balanço dos membros superiores, rigidez, marcha, tremor, face, fala e cuidados pessoais (WEBSTER, 1968); o Parkinson's Disease Questionnaire - 39 (PDQ-39), desenvolvido em 1995, com versão adaptada para o português (CAROD, MARTINEZ, VARGAS, 2007) e composto por 39 itens que avaliam mobilidade, atividade de vida diária, bem-estar emocional, estigma, apoio social, cognição, comunicação e desconforto corporal; o Parkinson's Disease Quality of Life Questionnaire (PDQL), criado em 1996, com versão adaptada para o português (CAMPOS, 2010), composto por 37 itens agrupados em quatro domínios relacionados a sintomas parkinsonianos, sintomas sistêmicos, função social e função emocional e a Escala de Atividade de Parkinson (PAS), desenvolvida em 2000 com o objetivo de caracterizar a independência funcional dos indivíduos através de 11 itens sobre transferências na cadeira, acinesia na marcha, mobilidade na cama e mobilidade na cama com uso do cobertor (NIEUWBOER et al., 2000). O questionário foi composto por 14 perguntas fechadas relacionadas a gênero, faixa etária, tempo de diagnóstico, medicamentos em uso e os sintomas motores mais comuns na DP, dentre eles, um sintoma não motor, a dor, devido à sua importância na prática fisioterapêutica.

Após a coleta, os dados foram exportados para um sistema de banco de dados, sendo analisados através de frequência e percentual com auxílio do programa Microsoft ${ }^{\circledR}$ Excel. 


\section{RESULTADOS}

Dos 30 parkinsonianos, 19 (63\%) eram do sexo masculino e 11 (37\%) do sexo feminino, com tempo de diagnóstico variando de 2 meses a 16 anos (média de \pm 6 anos). A média de idade dos participantes foi de 64 anos e em relação ao tratamento da doença, 24 (80\%) utilizavam o medicamento Prolopa.

Os sintomas motores dos participantes da pesquisa encontram-se na Tabela 1.

Tabela 1 - Prevalência de sintomas motores em portadores de Doença de Parkinson. Barra Mansa - RJ, Brasil, 2019.

\begin{tabular}{lll} 
Sintomas motores & N & $\%$ \\
\hline & & 87 \\
Bradicinesia & 26 & 73 \\
Instabilidade postural & 22 & 70 \\
Micrografia & 21 & 70 \\
Marcha com passos curtos & 21 & 63 \\
Rigidez muscular & 19 & 63 \\
Tremor & 19 & 60 \\
Câimbra & 18 & 57 \\
Congelamento (Freezing) & 17 & 47 \\
Redução ou ausência do balanço dos braços & 14 & 43 \\
Distonia & 13 & 43 \\
Hipofonia & 13 & 33 \\
Discinesia & 10 & \\
\hline
\end{tabular}

Fonte: Banco de dados dos autores (2019).

\section{DISCUSSÃO}

A média de idade da população do estudo foi de 64 anos, assim como no estudo de Silva et al. (2019), em que a maioria dos participantes eram idosos. Esses resultados corroboram com a literatura que considera a idade o principal 
fator de risco para a DP (TANG et al., 2016). Quanto ao gênero, a maioria dos indivíduos da amostra estudada era composta por homens. Este resultado é semelhante ao encontrado no estudo de Fernandes e Filho (2018), em que quase $70 \%$ da amostra analisada corresponderam a pacientes do gênero masculino. A explicação para esses achados está fundamentada na hipótese que sugere a ação neuroprotetora dos estrogênios, hormônios femininos, às células neuronais (DIAZGRANADOS SANCHEZ et al., 2011).

A bradicinesia foi relatada por $87 \%$ dos entrevistados, sendo o sintoma motor mais comum entre eles, assim como pôde ser verificado no estudo de Silva et al. (2010), em que o sintoma apareceu em $80 \%$ da amostra estudada. A literatura mostra que esse sintoma é considerado o mais incapacitante da DP. Sua principal característica é a lentidão dos movimentos, entretanto, afeta também a iniciação dos movimentos, amplitude e força muscular (MENTZEL et al., 2016).

Um sintoma não motor comum entre $77 \%$ dos participantes do estudo foi a dor. Em uma pesquisa realizada por Blood (2016), a dor foi relatada por $61,8 \%$ dos parkinsonianos, corroborando com os resultados do atual estudo. Scalzo et al. (2018), afirmam que pacientes com DP têm um aumento na sensibilidade à dor, quando comparados a indivíduos saudáveis. Segundo a revisão sistemática de Sung et al. (2018), a hiperalgesia é o mecanismo responsável por esse sintoma no paciente com DP. Segundo Umphered (2004), a dor pode se apresentar como câimbras em pacientes com DP, sendo este um sintoma motor mencionado por $60 \%$ dos participantes deste estudo.

Os entrevistados também relataram dificuldade de equilíbrio/instabilidade postural. O que se verificou também na análise de Silva et al. (2011), em que a mesma queixa estava presente na maioria dos voluntários do estudo. Isso pode ser justificado pelo comprometimento da habilidade do SNC em processar as aferências vestibulares, visuais e proprioceptivas, as quais são interpretadas pelo portador da DP para gerar as respostas musculares 
adequadas responsáveis pela manutenção do equilíbrio corporal, podendo levar a ocorrência de quedas (SAITO et al., 2011; LORD et al., 2017). De acordo com Barbosa et al. (2016), as pessoas com DP têm 62\% mais experiências com quedas, do que indivíduos com outras doenças neurológicas.

A marcha com passos curtos foi apresentada por 70\% dos indivíduos, sendo que em $47 \%$ dos entrevistados havia redução ou ausência do balanço dos braços. De acordo com Massano (2011), na DP a marcha é lenta, os passos são curtos e há redução do balanceio dos membros superiores. A literatura mostra que esses sintomas estão associados à progressão da bradicinesia e seu conhecimento e identificação são primordiais ao cuidado, pois, possibilitam que o profissional identifique precocemente os pacientes em risco de quedas (JEONGHO; KANG; HORAK, 2015).

Os dados apresentados mostram a presença de micrografia, ou seja, a redução do tamanho da letra, na maioria dos voluntários. De acordo com Poewe et al. (2017), esse sintoma motor está entre as manifestações clínicas secundárias da DP. Para Santos et al. (2018), a micrografia associada ao tremor de repouso, surgem como manifestação inicial da doença. A maioria dos participantes do estudo também apresenta tremor, sendo que em boa parte deles o sintoma ocorre durante o repouso. No estudo de Silva et al. (2011), dentre as manifestações motoras, o tremor de repouso foi o sintoma mais prevalente, correspondendo a $80 \%$ dos indivíduos avaliados, contrapondo os achados deste estudo. Segundo Haase, Machado e Oliveira (2017), a bradicinesia é um sinal que mais serve para diferenciar a DP de outras alterações motoras, entretanto, Jankovic (2016) afirma que o tremor é o sinal mais reconhecível da DP.

Percebeu-se que a rigidez muscular foi sintoma comum entre os integrantes da pesquisa. Fernandes e Filho (2018), em estudo, apontam que dentre os sinais e sintomas motores que os pacientes apresentaram durante o curso da doença, a rigidez apareceu em $81 \%$ da amostra estudada. Já no estudo 
de Silva et al. (2010), 100\% da amostra referiu-se à rigidez. Apesar de variar discretamente em proporção, os três estudos corroboram com os dados encontrados por Meneses e Teive (2003), que indicam que a DP é caracterizada, dentre outros sintomas, pela rigidez muscular.

O congelamento (freezing) foi citado por 57\% dos participantes, corroborando com o estudo de Silva et al. (2011), onde 60\% dos indivíduos da amostra analisada apresentaram o sintoma. Segundo Bekkers et al. (2018), essa é uma característica marcante da marcha parkinsoniana que ocorre mais frequentemente com a progressão da doença. Ela é definida como a ausência breve e episódica ou redução acentuada da progressão dos pés para frente, apesar da intenção do indivíduo em andar.

A distonia foi relatada por $43 \%$ dos participantes, sendo a maior parte referida como torção do pescoço e torção das costas. Segundo Letro (2007), a distonia é muito comum no pé do lado mais afetado pela DP. Quando sua manifestação ocorre pela manhã, se apresenta como inversão e equinismo doloroso, sendo, portanto, mais um sintoma motor que pode ocasionar dor.

A hipofonia foi observada em $43 \%$ dos indivíduos, o que não corrobora com o estudo de Fernandes e Filho (2018) no qual apenas 3,45\% apresentaram este sintoma. Conforme Ramig, Fox e Sapir (2004), as alterações da fala podem incidir em 50 a 90\% dos pacientes com DP. Em relação à discinesia, 33\% dos voluntários relataram o sintoma. Na análise de Spitz et al. (2017) foi observado que as discinesias apareceram em 13,68\% dos participantes. Dados da literatura mostram que as discinesias podem ser causadas pelas drogas antiparkinsonianas, como o Levodopa quando usado por extensos períodos (HAASE; MACHADO; OLIVEIRA, 2017). Segundo Rascol et al. (2015) e Jeongho, Kang, Horak (2015), a discinesia é a ocorrência de movimentos corporais involuntários que comprometem a mobilidade do indivíduo. 
Considera-se o tamanho amostral uma limitação do presente estudo, assim como o instrumento de avaliação, que pode ser aprimorado e complementado por outros métodos em novos estudos, com um número maior de parkinsonianos. Embora apresente limitações, observa-se uma importante implicação prática do estudo, já que seus resultados podem contribuir para uma tomada de decisão dos fisioterapeutas durante o processo de reabilitação.

\section{Considerações Finais}

Após a análise dos resultados, verificou-se que diversos são os comprometimentos motores apresentados pelos participantes do estudo, sendo a bradicinesia o sintoma mais comum entre eles, seguido da instabilidade postural, micrografia, marcha com passos curtos, rigidez muscular, tremor, câimbra, congelamento (freezing), redução ou ausência do balanço dos braços, distonia, hipofonia e discinesia. Associada aos sintomas motores, a dor foi um sintoma não motor identificado como o mais comum entre os entrevistados.

\section{Referências}

BARBOSA, A. F. et al. Marcha, postura e cognição na doença de Parkinson. Dement Neuropsychol, v. 4, n. 10, p. 280-286, 2016.

BEKKERS, E. M. J. et al. The Impact of Dual -Tasking on Postural Stability in People With Parkinson's Disease With and Without Freezing of Gait. Neurorehabilitation And Neural Repair, v. 32, n. 2, p.166 -174, 2018.

BLOOD, M. R. Y. Avaliação clínica da dor no período de wearing off de pacientes com doença de Parkinson em tratamento com levodopa. 2016. 91f. Dissertação (Mestrado em Fisiologia e Fisiopatologia) - Universidade Estadual de Ponta Grossa, Ponta Grossa, PR, 2016. 
CAMPOS, M. Tradução, Adaptação Cultural e Validação do Parkinson's Disease Quality of Life-questionnaire (PDQL) para o português falado no Brasil, o PDQL-BR. 2010. 81f. Dissertação (Mestrado em Ciências da Saúde) Faculdade de Medicina, Universidade Federal de Uberlândia, Uberlândia, 2010.

CAROD, A. F. J.; MARTINEZ, M. P.; VARGAS, A. P. Independent validation of SCOPA-Psychosocial and metric properties of the PDQ-39 Brazilian version. Movement Disorders, v. 22, n. 1, p. 91-98, 2007.

CANNING, C. G. et al. Exercise for falls prevention in Parkinson disease: a randomized controlled trial. Neurology, v. 84, n. 3, p.304-12, 2015.

CUGUSI, L. et al. Effects of a Nordic Walking program on motor and nonmotor symptoms, functional performance and body composition in patients with Parkinson's disease. NeuroRehabilitation, v. 37, n. 2, p. 245-54, 2015.

DAYAN, E; BROWNER, N. Alterações na conectividade funcional intrinsic striato-thalamo-pallidal como um pródomo da doença de Parkinson. Neurolmage: CLINICAL, n. 16, p. 1-9, 2017.

DIAZGRANADOS SÁNCHEZ, J. A. et al.. Descripción de la población de pacientes con enfermedad de Parkinson en un centro médico neurológico en la ciudad de Cali, Colombia. Acta Neurol Colomb, v. 27, n. 4, p. 205-9, 2011.

ERKKINEN, M. G.; OHK, K.; GESCHWIND, M. Neurologia Clínica e Epidemiologia das Doenças Neurodegenerativas Principais. Cold Spring Harb Perspect Biol, v. 10 n. 4, p. 1-46 2018.

FARIA, L. J. F.; LIMA, P. M. R.; PEREIRA-SILVA, N. L. Resiliência familiar diante do diagnóstico da doença de Parkinson na velhice. Revista Pesquisas e Práticas Psicossociais, v. 14, n. 1, p. 1-18, 2019.

FERNANDES, I.; FILHO, A. S. A. Estudo Clínico-Epidemiológico de pacientes com Doença de Parkinson em Salvador-Bahia. Revista Brasileira de Neurologia e Psiquiatria, v. 22, n. 1, p. 45-59, 2018. 
GONÇALVES, L. H. T.; ALVAREZ, A. M.; ARRUDA, M. C. Pacientes portadores da Doença de Parkinson: significado de suas vivências. Acta Paulista de Enfermagem, v. 20, n. 1, p. 62-68, 2007.

HAASE, D. C. B. V.; MACHADO, D. C.; OLIVEIRA, J. G. D. Atuação da Fisioterapia no paciente com Doença de Parkinson. Revista Fisioterapia em Movimento, v. 21, n. 1, p. 79-85, 2017.

HOEHN, M. M.; YAHR, M. D. Parkinsonism: onset, progression and mortality. Neurology, v. 17, n. 5, p. 427-442, 1967.

JANKOVIC, J. How Do I examine for re-emergent tremor? International Parkinson and Movement Disorder Society, v. 3, n. 2, p. 216-17, 2016.

JEONGHO, P.; KANG, Y.J.; HORAK, F. B. What Is Wrong with Balance in Parkinson's Disease? Journal Movement Disorders, v. 3, n. 8, p.109-114, 2015.

LETRO, G. H. Dor na Doença de Parkinson. 2007. 80f. Dissertação (Mestrado em Ciências Médicas) - Faculdade de Ciências Médicas da Universidade Estadual de Campinas, Campinas, SP, 2007.

LORD, S. et al. Natural history of falls in an incident cohort of Parkinson's disease: early evolution, risk and protective features. Neurology, v. 264, n. 11, p. 2268-2276, 2017.

MASCARENHAS, C. H. M.; SOUZA, M. P. Avaliação funcional de indivíduos portadores da doença de Parkinson. Arquivos de Ciências da Saúde, v. 17, n. 4, p. 179-184, 2010.

MASSANO, J. Doença de Parkinson: atualização clínica. Acta Médica Portuguesa, v. 24, n. 54, p. 827-834, 2011.

MENESES, M. S.; TEIVE, H. A. Ghizoni. Doença de Parkinson. Rio de Janeiro: Guanabara Koogan; 2003. 
MENTZEL, T. Q. et al. Instrumental Assessment of Bradykinesia: A Comparison Between Motor Tasks. IEEE Journal of Biomedical and Health Informatics, v. 20, n. 2, p. 521-526, 2016.

MINISTÉRIO DA SAÚDE. Resolução n 466, de 12 de dezembro de 2012. Diário Oficial da União, 13 de junho de 2013. Seção 1, p. 59.

MINISTÉRIO DA SAÚDE. Resolução n 510, de 07 de abril de 2016. Diário Oficial da União, 24 de maio de 2016. Seção 1, p. 46.

NIEUWBOER, A. et al. Development of an activity scale for individuals with advanced Parkinson's Disease: reliability and "on-off" variability. Physical Therapy, v. 80, n. 11, p. 1087-96, 2000.

PETERNELLA, F. N. M.; MARCON, S. S. Descobrindo a Doença de Parkinson: impacto para o parkinsoniano e seu familiar. Revista Brasileira Enfermagem, v. 62, n. 1 , p. 25-31, 2009.

POEWE, W. et al. Parkinson disease. Nature Reviews Disease Primers, v. 3, n. 1, p. 1-21, 2017.

RAMIG, L. O.; FOX, C.; SAPIR, S. Parkinson's disease: speech and voice disorders and their treatment with the Lee Silverman Voice Treatment. Semin Speech Lang, v. 25, n. 2, p. 169-180, 2004.

RASCOL, O. et al. Falls in ambulatory non-demented patients with Parkinson's disease. Journal Of Neural Transmission, v. 122, n. 10, p.1447-1455, 2015.

ROWLAND, L. P.; PEDLEY, T. A. M. Tratado de neurologia. 12ª ed. Rio de Janeiro: Guanabara Koogan; 2011.

SAITO, T. C. A Doença de Parkinson e seus tratamentos: uma revisão bibliográfica. 2011. 35f. Monografia (Especialização em Saúde Coletiva e Saúde da Família) - Centro Universitário Filadélfia, Londrina, PR, 2011. 
SANTOS, A. R. S. et al. Sintomas não-motores na doença de Parkinson: estudo qualitativo exploratório. 2018. 133f. Dissertação (Mestrado Integrado em Psicologia) - Universidade de Lisboa, Lisboa, 2018.

SCALZO, P. L. et al. Caracterização da dor em pacientes com doença de Parkinson. Revista Brasileira de Neurologia, v. 54, n. 4, p. 19-25, 2018.

SHULMAN, L. M. et al. Randomized clinical trial of 3 types of physical exercise for patients with Parkinson disease. Jama Neurology, v. 70, n. 2, p. 183-90, 2013.

SILVA, F. S. et al. Evolução da doença de Parkinson e comprometimento da qualidade de vida. Revista Neurociências, v. 18, n. 4, p. 463-8, 2010.

SILVA, P. F. C. Correlação entre perfil clínico, qualidade de vida e incapacidade dos pacientes da Associação Brasil Parkinson. ConScientiae Saúde, v. 10, n. 4, p. 650-656, 2011.

SILVA, P. C. S.; FERNANDES, A. C. B. C.; TERRA, F. S. Avaliação da depressão e da capacidade funcional em idosos com Doença de Parkinson. Revista de Enfermagem UFPE, v. 8, n. 7, p. 1920-7, 2014.

SILVA, R. M. et al. Prevalência de disfunção temporomandibular em pessoas com Parkinson em hospital público universitário. Revista CEFAC, v. 21, n. 3, p. 1-10, 2019.

SPITZ, M. et al. Análise dos sintomas motores na Doença de Parkinson em pacientes de hospital terciário do Rio de Janeiro. Revista Brasileira de Neurologia, v. 53, n. 3, p. 14-18, 2017.

TANG, Y. et al. Cerebral Metabolic Differences Associated with Cognitive Impairment in Parkinson's Disease. PLoS One, v. 11, n. 4, p. 1-11, 2016.

UMPHERED, D. A. Reabilitação neurológica. 4ª ed. São Paulo: Manole; 2004.

VERAS, R. P.; OLIVEIRA, M. Envelhecer no Brasil: a construção de um modelo de cuidado. Revista Ciência \& Saúde Coletiva, v. 23, n. 6, p. 1929-1936, 2018. 
VERAS, R. É possível, no Brasil, envelhecer com saúde e qualidade de vida?

Revista Brasileira de Geriatria e Gerontologia, v. 19, n. 3, p. 381-382, 2016.

WEBSTER, D. D. Critical analysis of the disability in Parkinson's disease.

Modern Treatment, v. 5, n. 2, p. 257-82, 1968. 